\title{
Delayed Cervical Vertebral Body Osteoradionecrosis in a Nasopharyngeal Carcinoma Patient - Case Report
}

\author{
Hsien-Ta Hsu ${ }^{1}$, Hung-Yu Li ${ }^{1}$, Stephen Shei-Dei Yang ${ }^{2,3}$ and Kuo-Feng Huang ${ }^{1,3^{*}}$ \\ ${ }^{1}$ Department of Surgery, Division of Neurosurgery, Taipei Tzu Chi Hospital, Buddhist Tzu Chi Medical Foundation, New Taipei City 23143, Taiwan \\ ${ }^{2}$ Department of Surgery, Taipei Tzu Chi Hospital, Buddhist Tzu Chi Medical Foundation, New Taipei City, Taiwan \\ ${ }^{3}$ School of Medicine, Tzu Chi University, Hualien 97004, Taiwan
}

"Corresponding author: Kuo-Feng Huang, Department of Surgery, Division of Neurosurgery, Taipei Tzu Chi Hospital, Buddhist Tzu Chi Medical Foundation, 289 Jianguo Rd, Xindian, New Taipei City 23143, Taiwan, Tel: +886-2-6628-9779; Fax: +886-2-6628-6255; E-mail: kuofeng1234@gmail.com

Received date: Mar 04, 2015, Accepted date: Mar 28, 2015, Publication date: Mar 30, 2015

Copyright: (c) 2015 Hsu HT, et al. This is an open-access article distributed under the terms of the Creative Commons Attribution License, which permits unrestricted use, distribution, and reproduction in any medium, provided the original author and source are credited.

\begin{abstract}
High dose radiotherapy is a treatment for nasopharyngeal carcinoma (NPC), but can result in radiation-induced changes to the cervical spine, which may be difficult to recognize. Pathological fractures occurring after radiation therapy for soft tissue tumors are rare. In this context, we report a rare case of delayed Osteoradionecrosis of a cervical vertebral body (C5) causing myeloradiculopathy. The patient, a 54-year-old man, received radical radiotherapy for NPC 10 years previously. Anterior decompression and stabilization with internal fixation was performed. Pathology reported necrotic tissue and no evidence of malignancy. Postoperatively, the patient's neck pain improved, and he recovery muscle power in all four extremities. Early diagnosis is essential for this curable condition. Emergency surgical intervention for decompression and stabilization with internal fixation may result in complete neurological recovery.
\end{abstract}

Keywords: Radiotherapy; Nasopharyngeal carcinoma; Osteoradionecrosis; Cervical vertebral body; Myeloradiculopathy

\section{Introduction}

Radiotherapy has important applications in a wide variety of neoplastic diseases, either as curative, adjuvant, or palliative therapy [1]. The effects of radiation on bone include growth disturbances, osteoradionecrosis (ORN) [2,3], and radiation-induced neoplasia[4,5]. A 5.6\% pathological fracture rate has been found after limb-conserving surgery and adjuvant radiation therapy for soft tissue tumors of the extremities [6]. There were also several reports of pelvic and sacral insufficiency fractures after irradiation for carcinoma of the cervix, endometrial carcinoma, and prostate carcinoma [7-9]. Nonetheless, to the best of our knowledge, there are no reports of osteoradionecrosis causing cervical vertebral body collapse. In this report, we describe a patient with delayed postirradiation necrosis causing cervical vertebral body collapse, leading to cervical radiculomyelopathy.

\section{Case Report}

The patient, a 54-year-old man, had a history of nasopharyngeal carcinoma (NPC), with right cervical lymph node metastasis. He underwent radiotherapy, (7,280 cGy/39 fractions). He completed the treatment smoothly, except for transient oral mucositis and a neck skin reaction. No gross tumor was visible on physical examination, and no residual lymph nodes were palpable in the right neck. He had been symptom free for 10 years.

The patient then suddenly suffered from severe neck and right upper extremity weakness for two weeks. Neurological examination showed that the right side deltoid and bicep muscle power had decreased (3/5) initially. The patient rapidly developed quadriparesis with a decrease in sensory level to below C5. Typical myelopathy was noted. A cervical spine X-ray showed C5 vertebral body collapse (Figure 1). Magnetic resonance imaging (MRI) showed low signal density on T1-weighted images, indicating vertebral collapse with cord compression at the level of C5 (Figure 2). Computed tomography (CT) revealed a pathological fracture of the vertebral body at the same level (Figure 3).

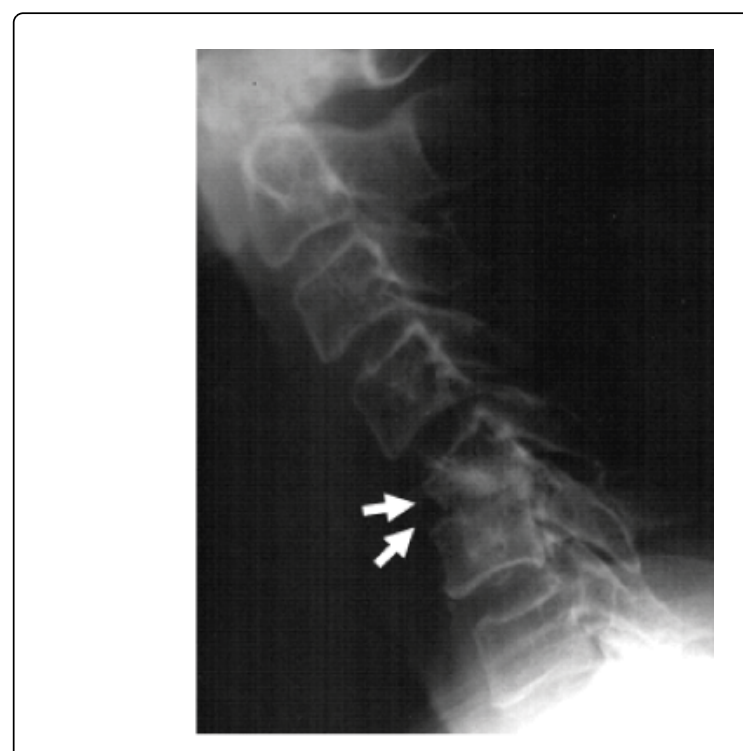

Figure 1: A cervical spine X-ray showed C5 vertebral body collapse. Arrows in the figures indicate the location of damage. 
Citation: Hsu HT, Li HY, Yang SSD, Huang KF (2015) Delayed Cervical Vertebral Body Osteoradionecrosis in a Nasopharyngeal Carcinoma Patient - Case Report. J Nucl Med Radiat Ther 6: 220. doi:10.4172/2155-9619.1000220

Page 2 of 3

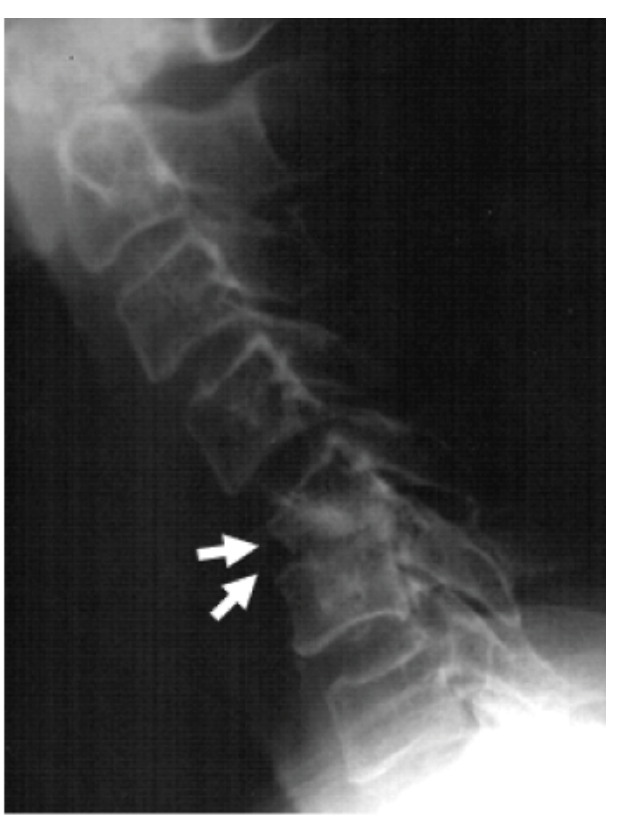

Figure 2: Magnetic resonance imaging (MRI) showed low signal density on T1-weighted images, indicating vertebral collapse with cord compression at the level of C5. Arrows in the figures indicate the location of damage.

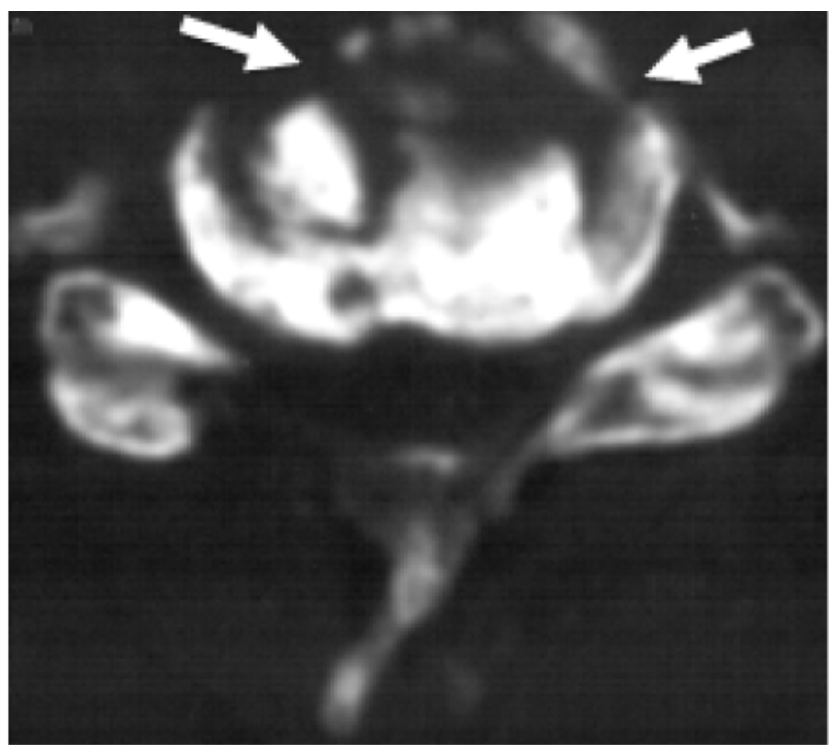

Figure 3: Computed tomography (CT) revealed a pathological fracture of the vertebral body at the same level. Arrows in the figures indicate the location of damage.

The initial differential diagnosis included tumor metastasis, radiation-necrosis, or radiation-induced malignant change. After the patient was placed in cervical traction, the muscle power of all extremities improved. Anterior C5 corpectomy and Caspar plate and screw fixation with allograft bone interbody fusion was performed.
Histologically (Hematoxylin-eosin stain), there was no evidence of malignant cells; only tissue necrosis associated with scattered, acute inflammatory cells was found (Figure 4).

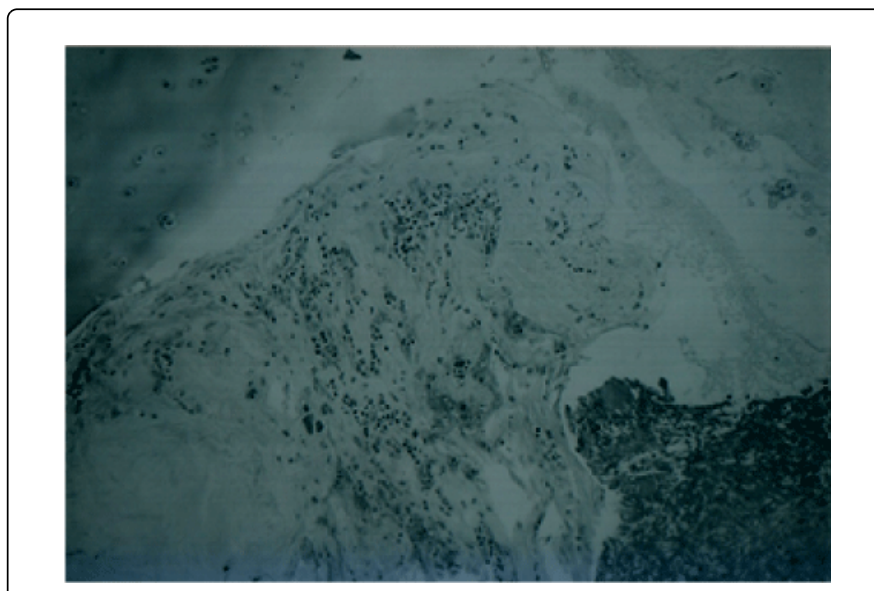

Figure 4: Histologically (Hematoxylin-eosin stain), there was no evidence of malignant cells; only tissue necrosis associated with scattered, acute inflammatory cells was found.

Postoperatively, the patient's neck pain improved, and a neurological examination showed recovery of muscle power in all four extremities. Nonetheless, mild weakness (4/5) in the right deltoid and biceps muscles persisted. After several weeks of rehabilitation, the patient resumed his premorbid level of activity and lifestyle.

\section{Discussion}

Radiation therapy is effective therapy for NPC. Nonetheless, radiation therapy may cause changes in the skeletal system. Complications in the mature skeleton include ORN, pathologic fractures and radiation-induced neoplasm $[1,4,5,9]$. Considerations for differential diagnosis include radiation induced sarcoma, recurring neoplastic disease and, of course, infection. Diagnosis of ORN can generally be made if bone changes are confined to the radiation fields, there is an absence of soft tissue mass and osseous alteration over time is stable [3,5].

Over the years, the criteria used to define ORN have varied. Gevorgyan and Wong et al defined ORN as the slow-healing, radiation-induced ischemic necrosis of bone associated with soft tissue necrosis of variable extent occurring in the absence of local primary tumor necrosis without recurrence of metastasis [10]. The effects of radiation in mature bone are dose related [11]. The threshold for radiation-induced changes in bone is $3,000 \mathrm{cGy}$, with cell death and devascularization of bone occurring at doses over 5,000 cGy [4]. The majority of cases of ORN occur in the mandible, clavicle, humeral head, ribs, and femur [4]. The specific radiographic findings vary with the site of involvement $[5,6,9,10]$.

The pathophysiology of ORN is believed to be a complex metabolic and homeostatic deficiency created by radiation-induced cellular injury and fibrosis, which is characterized by the formation of hypoxic, hypovascular and hypocellular tissue [3]. Radiation has been shown to cause a significant reduction in blood vessel formation at fracture sites [9]. Similarly, radiation can damage mature cortical bone by injuring the small Haversian vessels within the bone. Radiation also may have a 
Citation: Hsu HT, Li HY, Yang SSD, Huang KF (2015) Delayed Cervical Vertebral Body Osteoradionecrosis in a Nasopharyngeal Carcinoma

Page 3 of 3

direct toxic effect on primitive osteoradionecrosis cells [4]. The findings suggest the blood supply to the bone that would allow mesenchymal proliferation was impaired by the radiation $[1,3,4,6,9]$.

The most common presenting symptom of ORN is pain, regardless of the site involved $[1,2,4,10]$. In our patient, late collapse of a cervical vertebral body after tumor irradiation resulted in secondary kyphosis, causing anterior compression of the spinal cord. The patient initially presented with cervical radiculopathy, which rapidly progressed to quardriparesis. Although routine radiography may not show radiation-induced changes, sharp demarcation at the edge of the radiation port may be demonstrated with bone scanning or MRI in patients who have received doses as low as 3,600 cGy. Marrow changes in which hematopoietic cellular elements have been replaced with fat have high signal intensity on T1-weighted MR images and intermediate signal intensity on T2-weighted images [4,9]. T2weighted MRI may be useful in differentiating radiation fibrosis (hypointense) from recurrent tumor (typically hyperintense) [1,4]. These marrow changes occur as early as 2 months after radiation therapy and may be permanent $[4,8]$.

Treatment of ORN commonly requires the debridement of necrotic bone and hyperbaric oxygen (HBO) therapy [12]. The former removes any nidus for continued infection, while the latter increases tissue vascularity and improves healing [12]. Nonetheless, some studies show that surgery and $\mathrm{HBO}$ are not necessary for the resolution of ORN in selected patients [13]. In our patient, aggressive therapy to prevent or reverse the ulcer complications of cord compression helped to restore motor function and prevent further cord injury.

Patients who have undergone radiation therapy should subsequently be evaluated for radiation-induced changes. Routine follow-up imaging may show musculoskeletal complications or evidence of radiation therapy in asymptomatic patients. Symptomatic patients who have undergone radiation therapy for a primary neoplasm should be evaluated for recurrent or metastatic disease. Radiological assessment is the key in demonstrating characteristic patterns of radiation injury $[1,8,13]$. We emphasize that early diagnosis is essential for this curable condition. Awareness of the varied radiographic manifestations of radiation-induced changes in bone, and correlation with clinical features and the radiation field, will usually allow distinction of these changes from those associated with other pathologic conditions. Urgent surgical intervention for decompression and stabilization with internal fixation may result in complete neurological recovery.

\section{References}

1. Khan L, Tjong M, Raziee H, Lee J, Erler D, et al. (2015) Role of stereotactic body radiotherapy for symptom control in head and neck cancer patients.Support Care Cancer 23: 1099-1103.

2. Hillerup S, Elberg JJ, Thorn JJ, Andersen M (2014) Reconstruction of irradiated mandible after segmental resection of osteoradionecrosis-a technique employing a microvascular latissimus dorsi flap and subsequent particulate iliac bone grafting. Craniomaxillofac Trauma Reconstr 7: 190-196.

3. Rice N, Polyzois I, Ekanayake K, Omer O, Stassen LF (2015) The management of osteoradionecrosis of the jaws - A review.Surgeon 13: 101-109.

4. Mitchell MJ, Logan PM (1998) Radiation-induced changes in bone.Radiographics 18: 1125-1136.

5. Pateder DB, Eliseev RA, O'Keefe RJ, Schwarz EM, Okunieff P, et al. (2001) The role of autocrine growth factors in radiation damage to the epiphyseal growth plate.Radiat Res 155: 847-857.

6. Schoenfeld GS, Morris CG, Scarborough MT, Zlotecki RA (2006) Adjuvant radiotherapy in the management of soft tissue sarcoma involving the distal extremities.Am J Clin Oncol 29: 62-65.

7. Igdem S, Alco G, Ercan T, Barlan M, Ganiyusufoğlu K, et al. (2010) Insufficiency fractures after pelvic radiotherapy in patients with prostate cancer. Int J Radiat Oncol Biol Phys 77: 818-823.

8. Kim HJ, Boland PJ, Meredith DS, Lis E, Zhang Z, et al. (2012) Fractures of the sacrum after chemoradiation for rectal carcinoma: incidence, risk factors, and radiographic evaluation.Int J Radiat Oncol Biol Phys 84: 694-699.

9. Ugurluer G, Akbas T, Arpaci T, Ozcan N, Serin M (2014) Bone complications after pelvic radiation therapy: evaluation with MRI.J Med Imaging Radiat Oncol 58: 334-340.

10. Gevorgyan A, Wong K, Poon I, Blanas N, Enepekides DJ, et al. (2013) Osteoradionecrosis of the mandible: a case series at a single institution.J Otolaryngol Head Neck Surg 42: 46.

11. Kovacs CJ, Evans MJ, Daly BM (2003) Murine hematopoietic stem cell and stromal responses to clinically-related, fractionated radiotherapy (FxRT).Anticancer Res 23: 2625-2631.

12. Lim AA, Karakla DW, Watkins DV (1999) Osteoradionecrosis of the cervical vertebrae and occipital bone: a case report and brief review of the literature.Am J Otolaryngol 20: 408-411.

13. Kosaka Y, Okuno Y, Tagawa Y, Ueki N, Itoh K, et al. (2010) Osteoradionecrosis of the cervical vertebrae in patients irradiated for head and neck cancers.Jpn J Radiol 28: 388-394. 